\title{
RESPOSTAS CARDIOVASCULARES AGUDAS EM SESSÕES DE EXERCÍCIO RESISTIDO COM ESTIIMULOS DIFERENTES
}

\section{Acute Cardiovascular Responses to Resistance Training With Different Stimulus}

\author{
Carlos Eduardo Cardoso ${ }^{1}$ - cadumaumau@hotmail.com; Ricardo Aurélio Carvalho Sampaio² - ricardo@kuhp.kyoto-u.ac.jp \\ Thales Boaventura Rachid Nascimento 3 - boaventura.thales@gmail.com; Thiago Darci Dezingrini ${ }^{4}$ - thiagodd10@gmail.com \\ ${ }^{1}$ Centro Universitário EuroAmericano - Brasília - DF - Brasil. - 2Kyoto University - Kyoto - Japão - Graduate School of Medicine, \\ Department of Human Health Sciences. - ${ }^{3}$ Universidade Católica de Brasília - Brasília - DF - Brasil - \\ Programa de Pós-graduação Stricto Sensu em Educação Física. - ${ }^{4}$ Faculdade Alvorada - Brasília - DF - Brasil.
}

\begin{abstract}
Resumo: No exercício resistido (ER), a hipertrofia muscular pode ser estimulada pela tensão (caracterizados pelo uso de cargas elevadas, descansos maiores entre as séries e ênfase na fase excêntrica da contração) e pelas mudanças metabólicas (cargas e períodos de descansos menores, e maior número de repetições por série) impostas à fibra muscular. Assim, o objetivo deste estudo foi comparar em adultos jovens, as respostas agudas de frequência cardíaca (FC), pressão arterial sistólica (PAS), pressão arterial diastólica (PAD) e duplo produto (DP) entre duas sessões de ER, uma com característica tensional e outra metabólica. Quatorze homens $(24,1 \pm 3,43$ anos; $172,9 \pm 5,13 \mathrm{~cm} ; 77,6 \pm 7,9$ kg; gordura relativa $=15,2 \pm 4,3 \%$; força relativa $=5 \pm 1,1$ ), normotensos, aparentemente saudáveis e fisicamente ativos no ER foram submetidos à duas sessões de treinamento, ambas compostas por 3 séries realizadas no Leg Press $45^{\circ}$. A primeira sessão foi a metabólica (15 repetições com 2 seg para cada fase, $50 \%$ da carga máxima e 1 min de descanso) e 72 horas após, a tensional (6 repetições com 4 seg para a fase excêntrica e 2 seg para a concêntrica, $70 \%$ da carga máxima e 2 min de descanso). Por meio do Test " $t$ " de Student não-pareado ( $p<0,05)$, evidenciou-se que a sessão metabólica apresentou FC, PAS e DP significativamente maiores do que a tensional, sem diferença para a PAD. Em comparação à sessão metabólica, a tensional promove menor sobrecarga cardiovascular.
\end{abstract}

Palavras-Chave: Exercício resistido; Pressão arterial; Frequência cardíaca; Duplo produto.

Abstract: In the resistance training (RT), muscle hypertrophy can be stimulated by tension (characterized by the combination of heavier loads, longer rest period between sets and emphasis on the eccentric phase of contraction) and metabolic (lower loads, shorter rest periods and more repetitions per set) changes imposed to muscle fiber. Thus, the purpose of this study was to compare, in young adults, acute heart rate (HR), systolic blood pressure (SBP), diastolic blood pressure (DBP) and rate-pressure product (RPP) response between two sessions of RT, one with tension and other with metabolic characteristics. Fourteen men $(24,1 \pm 3,43$ years; $172,9 \pm 5,13 \mathrm{~cm} ; 77,6 \pm 7,9 \mathrm{~kg}$; relative body fat $=15,2 \pm 4,3$ $\%$; relative strength $=5 \pm 1,1$ ), normotensive, apparently healthy and physically active in RT underwent two training sessions, both composed of 3 sets performed at $45^{\circ}$ Leg Press. The first session was the metabolic (15 repetitions per set; 2 seconds for concentric and eccentric phases, $50 \%$ of one-repetition maximum and 1 min of rest) and 72 hours later; the tension session was performed ( 6 repetitions per set; 2 seconds for the concentric phase and 4 seconds to the eccentric phase; $70 \%$ of one-repetition maximum and 2 min rest). Through the Student's unpaired "t" Test $(p<0.05)$, the metabolic session elicited greater increase $(p<0,05)$ in HR, SBP and RPP than the tension session, with no difference for DBP. The tension session promotes a lower cardiovascular overload than the metabolic session.

Keywords: Resistance training, Blood pressure, Heart rate, Rate-Pressure Product.

Aceito em: 25/02/2011 - Revista de Educação Física 2012 Ago; 155:3-8. Rio de Janeiro - Brasil

\section{INTRODUÇÃO}

A sobrecarga imposta ao sistema cardiovascular, durante o exercício resistido (ER), tem sido investigada por meio de variáveis como a frequência cardíaca ( $F C)$, pressão arterial(1,2,3,4,5,6,7) e duplo produto (DP) $)^{(1,4,5,7)}$ sendo este último, o melhor indicador indireto do trabalho cardíaco durante o $E R^{(8)}$, o que o credencia como uma importante variável na prescrição do $\mathrm{ER}^{(9)}$.

As respostas cardiovasculares durante a prática do ER são dependentes da carga mobilizada, do número de repetições realizadas ${ }^{(2,3)}$, do tipo de contração muscular(10) do tempo de descanso entre as séries ${ }^{(2,7)}$, da velocidade de execução(6), bem como da posição do corpo durante a execução do exercício(4).

Durante o treino de ER, a hipertrofia muscular pode ser estimulada pela tensão e pelas mudanças metabólicas impostas à fibra muscular. Os estímulos que priorizam a tensão são dados pela combinação do uso de cargas mais elevadas, descansos maiores entre as séries e ênfase na fase excêntrica da contração. Já os estímulos que priorizam as mudanças metabólicas, 
fazem a combinação de cargas e períodos de descansos menores, e maior número de repetições por série(11). Apesar disso, nenhum estudo que tenha analisado as respostas cardiovasculares dessas variações de treino foi encontrado.

Diante do exposto, e em razão do ER ser recomendado como parte integrante de programas de exercícios físicos para várias populações(8), faz-se relevante identificar os protocolos de ER que sejam capazes de estimular a hipertrofia muscular com menor sobrecarga ao sistema cardiovascular. Nesse sentido, o objetivo do presente estudo foi comparar em adultos jovens, as respostas agudas de FC, pressão arterial e DP entre duas sessões de ER, uma com característica tensional e outra metabólica.

\section{METODOLOGIA}

Estudo do tipo cruzado (crossover) com abordagem quantitativa. Quatorze homens com idade entre 19 e 30 anos, normotensos, aparentemente saudáveis e fisicamente ativos há pelo menos seis meses na modalidade de musculação participaram desse estudo. Os critérios de exclusão foram pressão arterial de repouso elevada, limitação motora, uso frequente (últimos 30 dias) de cafeína, álcool, nicotina e/ou esteróides anabolizantes. Todos os sujeitos foram voluntários e assinaram um termo de consentimento livre e esclarecido (TCLE), conforme Resolução no 196/96 do Conselho Nacional de Saúde do Brasil (12). O estudo foi aprovado por comitê de ética institucional.

Os dados foram coletados em três visitas não consecutivas para cada indivíduo (Figura 1). Na primeira visita, os voluntários assinaram o TCLE e responderam ao questionário de prontidão para a atividade física $(P A R-Q)^{(8)}$. Em seguida foram mensuradas as variáveis antropométricas e a carga máxima para o exercício Leg Press $45^{\circ}$.

Para mensuração da massa corporal utilizou-se uma balança mecânica (Filizola $\left.{ }^{\circledR}\right)$ e para a estatura um estadiômetro acoplado à balança. A gordura corporal relativa foi estimada por meio da técnica de dobras cutâneas, utilizando-se o compasso (Lange $®$ ) e o protocolo de sete dobras para homens ${ }^{(13)}$.

Conforme protocolo do $\mathrm{ACSM}^{(8)}$, foi realizado, no aparelho Leg press $45^{\circ}$ (Cybex $\left.\AA\right)$, o teste de uma repetição máxima (1RM) para mensurar a força máxima dos membros inferiores. A força relativa $(F R)$ foi calculada a partir da fórmula $F R=$ Carga $1 \mathrm{RM}(\mathrm{kg})$ / massa corporal $(\mathrm{kg})$. Para a realização do teste, o indivíduo sentou-se em um banco com inclinação de $45^{\circ}$, os pés foram posicionados na plataforma do aparelho em afastamento lateral, com os joelhos em flexão de $90^{\circ}$. Em seguida, realizouse uma extensão da articulação do quadril e do joelho simultaneamente, retornando à posição inicial para uma nova repetição. Para evitar a manobra de valsalva, os voluntários foram instruídos a expirar na fase concêntrica e a inspirar na excêntrica.

Na segunda visita (48 horas após a primeira), os voluntários tiveram sua FC e pressão arterial de repouso mensurada. Tais medidas foram obtidas após os voluntários permanecerem sentados por 10 minutos em local calmo. Em seguida, foi realizado um aquecimento de cinco minutos em cicloergômetro, com uma carga suficiente para elevar a FC entre 110 e 120 batimentos por minuto. Imediatamente após, os voluntários foram submetidos ao protocolo de ER com características metabólicas.

A FC foi mensurada por meio de um frequencímetro (Polar $\AA, R S 800$ sd) e a pressão arterial pelo método auscultatório, utilizando-se de um esfigmomanômetro coluna de mercúrio e um estetoscópio (Oxigel囚). A mensuração foi realizada no braço direito. O DP foi obtido da multiplicação da FC pela PAS.

$\mathrm{Na}$ terceira visita, com um intervalo de 72 horas da sessão metabólica, a FC e a pressão arterial em repouso também foram mensuradas, seguidas do aquecimento de cinco minutos (mesmo procedimento realizado na segunda visita) e foi realizado o protocolo com características tensionais. No período de coleta dos dados os voluntários foram instruídos a não realizarem exercícios físicos nos intervalos entre as sessões.

FIGURA 1 - CRONOGRAMA DA COLETA DE DADOS.

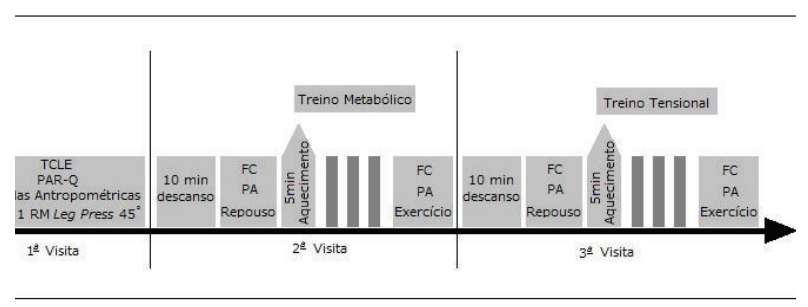

Para os valores em exercício, em ambos os 
protocolos, a pressão arterial foi mensurada durante a antepenúltima e última repetição ${ }^{(9,14)}$ sendo considerada a FC nesse mesmo momento. Com o propósito de se registrar os maiores valores, a pressão arterial e a FC foram mensuradas, somente, ao final da terceira série do exercício.

\section{Protocolo de treino}

Os dois protocolos de treinamento foram compostos por três séries, realizadas no aparelho Leg Press $45^{\circ}$ (Cybex®), seguindo o mesmo padrão de execução do teste de 1RM. Esse número de séries foi escolhido em razão de ser frequentemente utilizado em clubes e academias. Os protocolos de treinos foram elaborados conforme proposta de Gentil (11) (Quadro 1).

\begin{tabular}{|c|c|}
\hline \multicolumn{2}{|c|}{$\begin{array}{c}\text { QUADRO } 1 \text { - CARACTERÍSTICAS DOS PROTOCOLOS } \\
\text { DE EXERCÍCIO RESISTIDO. }\end{array}$} \\
\hline $\begin{array}{l}\text { Protocolo com característica } \\
\text { metabólica }\end{array}$ & $\begin{array}{l}\text { Protocolo com característica } \\
\text { tensional }\end{array}$ \\
\hline 15 repetições por série & 6 repetições por série \\
\hline $50 \%$ de $1 \mathrm{RM}$ & $70 \%$ de $1 \mathrm{RM}$ \\
\hline $\begin{array}{l}2 \text { segundos para a fase } \\
\text { concêntrica }\end{array}$ & $\begin{array}{l}2 \text { segundos para a fase } \\
\text { concêntrica }\end{array}$ \\
\hline $\begin{array}{l}2 \text { segundos para a fase } \\
\text { excêntrica }\end{array}$ & $\begin{array}{l}4 \text { segundos para a fase } \\
\text { excêntrica }\end{array}$ \\
\hline $\begin{array}{l}1 \text { minuto de descanso entre } \\
\text { as séries }\end{array}$ & $\begin{array}{l}2 \text { minutos de descanso } \\
\text { entre as séries }\end{array}$ \\
\hline
\end{tabular}

O volume total de treino de cada voluntário, em cada um dos protocolos de treino foi calculado: Volume total $(\mathrm{Kg})=$ Número de séries $\mathrm{x}$ Número de repetições $\mathrm{x}$ Carga levantada $(\mathrm{Kg})$. $O$ tempo sob tensão também foi calculado: Tempo sob tensão $(\mathrm{seg})=$ Tempo em cada série (seg) x Número de séries.

Os critérios adotados para a interrupção de ambos os protocolos de treinamento foram: dor no peito, queda (20 $\mathrm{mm} \mathrm{Hg}$ ) ou aumento excessivo da PAS > $260 \mathrm{~mm} / \mathrm{Hg}$ ou PAD $>115 \mathrm{~mm} / \mathrm{Hg}$, tontura, palidez, cianose, náusea ou solicitação de interrupção por parte do voluntário.

\section{Tratamento estatístico}

Para caracterizar a amostra e verificar a normalidade dos dados, foi utilizada a estatística descritiva e o teste de Shapiro-Wilk, respectivamente. O teste de Mann-Whitney foi empregado para comparar o tempo total sob tensão e o volume total de treino entre as sessões. O Test " $\mathrm{t}$ " de
Student para amostras não-pareadas foi utilizado para a comparação entre os treinos metabólicos e tensionais. O nível de significância considerado foi de $5 \%(p<0,05)$. Para análise dos dados foi utilizado o software Statistical Package for the Social Science (SPSS), versão 10.0.

\section{RESULTADOS}

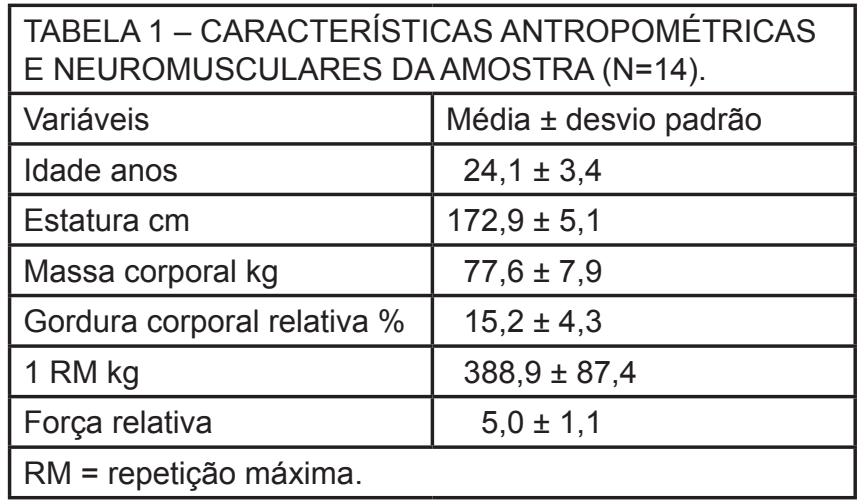

Em repouso pré-exercício, os valores da FC, PAS, PAD e DP não diferiram $(p>0,05)$ entre os dois protocolos de treino (Tabela 2).entanto, não foi observada diferença significativa entre as variáveis $(F 2=0,88 ; p=0,67$ ).

\begin{tabular}{|c|c|c|c|}
\hline \multicolumn{3}{|c|}{$\begin{array}{c}\text { Tabela 2 - Comparação da FC, PAS, PAD e DP em } \\
\text { repouso pré-exercício, entre o protocolo de treino } \\
\text { metabólico e tensional }(\mathrm{n}=14) .\end{array}$} \\
\cline { 1 - 3 } Variáveis & Metabólico & Tensional & $\mathrm{p}$ \\
\cline { 2 - 3 } & $\begin{array}{c}\text { Média } \pm \\
\text { desvio } \\
\text { padrão }\end{array}$ & $\begin{array}{c}\text { Média } \pm \\
\text { desvio } \\
\text { padrão }\end{array}$ \\
\hline FC bpm & $57,6 \pm 4,6$ & $58,6 \pm 5,7$ & 0,612 \\
\hline PAS mmHg & $128,6 \pm 11,8$ & $126,6 \pm 10,3$ & 0,649 \\
\hline PAD mmHg & $71,9 \pm 12,2$ & $70,9 \pm 12,6$ & 0,822 \\
\hline DP bpm/ & $7409,2 \pm$ & $7430,3 \pm$ & 0,957 \\
mmHg & 985,2 & 1043,6 & \\
\hline FC = freqüência cardíaca; PAS = pressão arterial sistólica; \\
PAD = pressão arterial diastólica; DP = duplo produto. \\
\hline
\end{tabular}

Entre os treinos executados, o protocolo com características metabólicas apresentou valores de FC, PAS e DP maiores do que o tensional $(p<0,05)$, sem diferença para os valores de PAD ( $p>0,05)$ (Tabela 3$)$. 


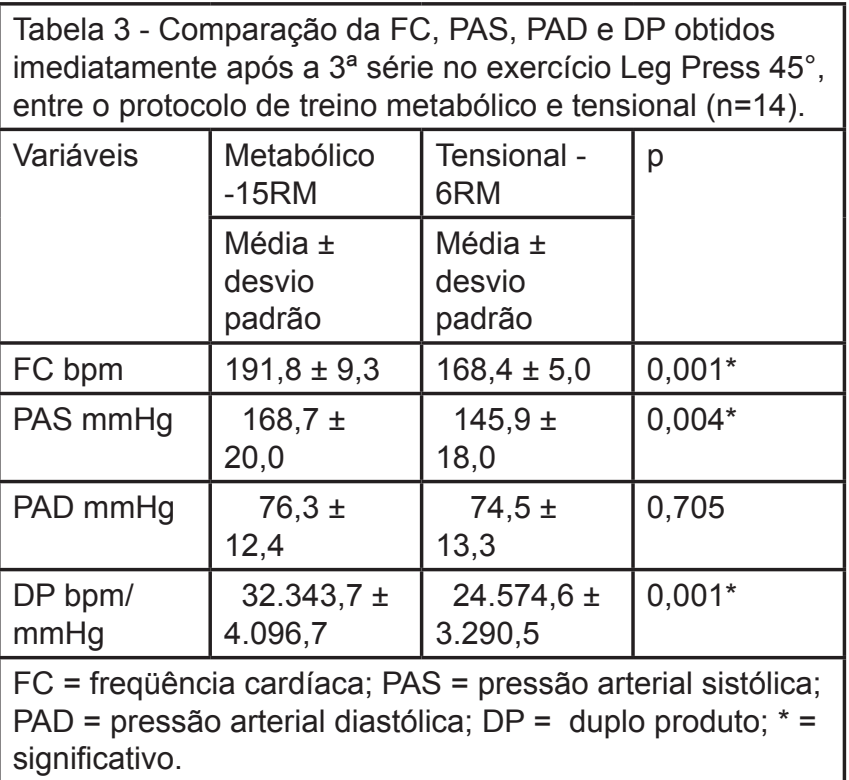

O volume total do protocolo de treino metabólico $(8.749,3 \pm 1.967,1 \mathrm{~kg})$ não diferiu $(p>0,05) \mathrm{em}$ comparação ao treino tensional $(9.799,2 \pm 2.203,2 \mathrm{~kg})$. Já o tempo sob tensão do protocolo metabólico (180 seg) foi maior $(p<0,05)$ em comparação ao tensional (108 seg).

\section{DISCUSSÃO}

Em repouso pré-exercício, a FC, a PAS, a PAD e o DP não diferiram entre as duas sessões de treino (Tabela 2). Esses achados sugerem que os voluntários encontravam-se em condições hemodinâmicas semelhantes em ambos os dias, bem como que o intervalo entre as duas sessões foi suficiente para que os efeitos agudos da primeira não interferissem sobre as variáveis cardiovasculares da segunda.

O protocolo tensional, o qual foi desempenhado com maiores cargas, menor número de repetições e descansos mais longos entre as séries, apresentou menores valores de FC, PAS e DP (Tabela 3). Concordante $\mathrm{a}$ isso, pesquisadores que avaliaram a execução do exercício cadeira extensora ${ }^{(3,14)}$ e Leg Press $^{(7)}$ realizado com maior carga e menor número de repetições, evidenciaram menores valores de $\mathrm{FC}^{(3,7)}$, PAS ${ }^{(3,7,14)}$ e $\mathrm{DP}(7)$ do que o realizado com menor carga e maior número de repetições, sendo que períodos de descanso mais longos entre as séries promoveram, em relação aos mais curtos, menor elevação da FC, PAS ${ }^{(2,7)}$ e DP (7). Apesar disso, há pesquisadores que evidenciaram respostas cardiovasculares semelhantes entre as séries realizadas com 6 e 12 repetições máximas ${ }^{(1)}$.

No que se refere às fases da contração muscular, o protocolo de ER tensional enfatizou, em relação ao metabólico, a fase excêntrica da contração e exibiu menor resposta cardiovascular. Indo ao encontro desse achado, o exercício agachamento(10), composto somente pela fase excêntrica da contração, promoveu, em relação aquele composto somente pela fase concêntrica, menores respostas cardiovasculares. Essa mesma relação foi evidenciada em exercício desempenhado com pequenos grupamentos musculares ${ }^{(15)}$.

A fase concêntrica da contração muscular promove, em relação à excêntrica, maior consumo de oxigênio. Ademais, protocolos deERcomênfasenafaseconcêntrica (16) promovem maiores concentração de lactato(17), o qual estimula o sistema nervoso simpático(18). Adicionado a isso, uma maior demanda metabólica é observada em protocolos de ER com menores intervalos de descanso entre as séries ${ }^{(19)}$. Desse modo, a presença desses dois fatores no treino metabólico pode ter promovido, em comparação ao tensional, maior estimulação simpática e por consequência, maior resposta da FC, PAS e DP. Outro fator que pode ter contribuído para as diferenças entre os protocolos de ER é o fato da fase concêntrica, a qual foi enfatizada no treino metabólico, promover redução do fluxo sanguíneo via oclusão vascular mecânica(20). Por consequência, há aumento da resistência vascular e das variáveis hemodinâmicas.

No que se refere à PAD, essa não diferiu entre os dois protocolos de ER (Tabela 3), tal como evidenciado por outros pesquisadores ${ }^{(10)} \mathrm{em}$ que a resposta dessa variável foi semelhante entre o agachamento realizado somente com contrações concêntricas e excêntricas. Ademais, a PAD obtida imediatamente após o ER foi muito próximo aos valores de repouso, tal como em evidenciado por outros pesquisadores $(4,5)$ que se valeram do método auscultatório.

Os valores de pressão arterial diminuem rapidamente ao término do $E R{ }^{(2)}$. Ademais, o método auscultatório é relativamente demorado(9). Portanto, é possível que ao se ouvir o $\mathrm{V}$ som de korotkoff (último som audível na aferição da PA), a PAD já tenha retornado aos valores próximos de repouso. Por consequência, os valores de PAD registrados foram próximos aos de repouso e semelhantes entre os métodos.

Como limitação, o presente estudo apresenta a 
utilização de somente um aparelho de musculação, haja vista que, o aparelho utilizado e a posição do corpo durante a execução do exercício(4) influenciam as respostas cardiovasculares. Além disso, o método aqui empregado para mensurar a pressão arterial foi o auscultatório, o qual subestima os valores pressóricos ${ }^{(14)}$.

Outro fator que poderia ser apontado como limitação, é o maior tempo sob tensão durante o protocolo metabólico em comparação ao tensional. Porém, há estudos em que um maior tempo sob tensão não resultou em uma maior resposta da $\mathrm{FC}$, pressão

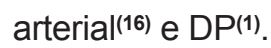

\section{CONCLUSÃO}

Em homens saudáveis, o protocolo de treino com características tensionais, executado no aparelho Leg Press $45^{\circ}$, promove menor resposta da FC, PAS e DP, quando comparado ao protocolo de treino metabólico. Portanto, em relação aos parâmetros cardiovasculares, o treino tensional parece ser mais seguro do que o metabólico, o que o credencia como método de ER mais viável para populações em que a sobrecarga cardíaca deva ser minimizada. Entretanto, estudos futuros com outras populações e exercícios devem ser realizados a fim de confirmar os resultados aqui encontrados.

\section{REFERÊNCIAS BIBLIOGRÁFICAS}

1. Lucas L, Farinatti PTV. Influência da carga de trabalho e tempo de tensão sobre as respostas agudas de freqüência cardíaca, pressão arterial sistólica e duplo-produto durante exercícios contra-resistência em mulheres idosas. Revista Brasileira de Ciência e Movimento 2007;15:75-82.

2. Lamotte $M$, Fournier $F$, Vanissium A, Van de borne $P$. Influence of rest period duration between successive muscular strength sets on acute modifications of blood pressure and heart rate in the healthy subject. Isokinet Exerc Sci 2006;14:349-55.

3. Lamotte M, Niset G, Van de borne P. The effect of different intensity modalities of resistance training on beat-to-beat blood pressure in cardiac patients. Eur $\mathrm{J}$ Cardiovasc Prev Rehabil 2005;12:12-7.

4. Miranda H, Simão R, Lemos A, Dantas BHA, Baptista LA, Novaes J. Análise da freqüência cardíaca, pressão arterial e duplo-produto em diferentes posições corporais nos exercícios resistidos. Rev Bras Med Esporte 2005;11:295-8.
5. D’Assunção W, Daltro M, Simão R, Polito M, Monteiro W. Acute cardiovascular responses in strength training conducted in exercises for large and small muscular group. Rev Bras Med Esporte 2007;13:118-122.

6. Wickwire PJ, McLester JR, Green JM, Crews TR. Acute heart rate, blood pressure, and RPE responses during super slow vs. traditional machine resistance training protocols using small muscle group exercises. J Strength Cond Res 2009;23:72-9.

7. Castinheiras-Neto AG, Da Costa-Filho IR, Farinatti PTV. Respostas cardiovasculares ao exercício resistido são afetadas pela carga e intervalos entre séries. Arq Bras Cardiol 2010;95:493-501.

8. Diretrizes do American College of Sports Medicine para os testes de esforço e sua prescrição. Rio de Janeiro: Guanabara Koogan; 2007.

9. Polito MD, Farinatti PTV. Considerações sobre a medida da pressão arterial em exercícios contraresistência. Rev Bras Med Esporte 2003;9:25-33.

10. Vallejo AF, Schroeder ET, Zheng L, Jensky NE, Sattler FR. Cardiopulmonary responses to eccentric and concentric resistance exercise in older adults. Age and Ageing 2006;35:291-7.

11. Gentil PRV. Bases científicas do treinamento de hipertrofia. Rio de Janeiro: Sprint; 2005.

12. BRASIL. Conselho Nacional de Saúde. Brasil. Resolução no 196/out., 1996. Disponível em: http:// conselho.saude.gov.br (19 jul. 2010).

13. Jackson AS, Pollock ML. Generalized equations for predicting body density of men. Br J Nutr 1978;40:497504.

14. Polito MD, Farinatti PTV, Lira VA, Nobrega ACL. Blood pressure assessment during resistance exercise: comparison between auscultation and Finapres. Blood Pressure Monitoring 2007;12(2):81-86.

15. Okamoto T, Masuhara M, Ikuta K. Relationship between plasma endothelin-1 concentration and cardiovascular responses during high-intensity eccentric and concentric exercise. Clin Physiol Funct Imaging 2008; 28:43-48.

16. Meyer K, Steiner R, Lastayo P, Lippuner K, Allemann Y, Eberli F, Schmid J, Saner H, Hoppeler H. Eccentric exercise in coronary patients: central hemodynamic and metabolic responses. Med Sci Sports Exerc. 2003;35:1076-82. 
17. Goto K, Ishii N, Kizuka T, Kraemer RR, Honda Y, Takamatsu K. Hormonal and metabolic responses to slow movement resistance exercise with different durations of concentric and eccentric actions. Eur J Appl Physiol 2009;106:731-9.

18. Cui J, Mascarenhas V, Moradkhan R, Blaha C, Sinoway LI. Effects of muscle metabolites on responses of muscle sympathetic nerve activity to mechanoreceptor(s) stimulation in healthy humans. Am J Physiol Regul Integr Comp Physiol. 2008;294:458-66.

19. Ratamess NA, Falvo MJ, Mangine GT, Hoffman JR, Faigenbaum AD, Kang J. The effect of rest interval length on metabolic responses to the bench press exercise. Eur J Appl Physiol 2007;100:1-17.

20. Mcardle WD, Katch FI, Katch VL. Fisiologia do exercício: energia, nutrição e desempenho humano. Rio de Janeiro: Guanabara Koogan; 2008.

Endereço para correspondência:

Fabrício Vieira do Amaral Vasconcellos

fabriciovav@hotmail.com

2178328455 / 2124274450

Rua Edgard Werneck, n. 131 bl. 03 c. 75/102 -

Jacarepagua - Rio de Janeiro - RJ - CEP 22763-010 